\title{
Segmentation of Laparoscopic Images for Computer Assisted Surgery
}

\author{
Jonathan Boisvert ${ }^{1}$, Farida Cheriet $^{1}$, and Guy Grimard ${ }^{2}$ \\ 1 École Polytechnique de Montréal, P.O. Box 6079, Station Centre-Ville, Montréal \\ H3C 3A7, Canada \\ \{jonathan.boisvert, farida.cheriet\} @polymtl.ca \\ 2 Hôpital Sainte-Justine, 3175 chemin de la côte Sainte-Catherine \\ Montréal H3T 1C5, Canada \\ guy_grimard@ssss.gouv.qc.ca
}

\begin{abstract}
This paper presents a learning-based approach to the problem of segmentation of laparoscopic images. The first step of the proposed method is to preprocess input images with a homomorphic filter. An initial segmentation map is then computed using a region growing based image segmentation algorithm. The obtained regions are finally classified using a support vector machine (SVM) to produce the final segmentation. The preliminary results computed on two image sets were promising. The first set includes laparoscopic images recorded in a controlled environment. The second set includes laparoscopic images recorded during three disk removal surgeries performed laparoscopically at Sainte-Justine Hospital.
\end{abstract}

\section{Introduction}

Laparoscopy is a relatively new surgical intervention that has been gaining in popularity in the medical community over the years. When performing a laparoscopic intervention instead of traditional surgery, the surgeon makes only small incisions in the patient's body and inserts camera, light source and medical instruments through the incisions to perform the surgical procedure using only a camera display instead of direct visualization. The advantages of this technique include small incisions, less blood loss, reduced post-operative pain, earlier discharge from the hospital and faster post-operative rehabilitation.

Economical and human advantages are great; however, the limited visualization offered to the surgeon is a source of difficulties. For example, because the field of view of the endoscope is small, the surgeon lacks information about the relative context of the endoscope and its surroundings. This information is especially important to keep track of the position of sensitive anatomical structures such as the azygos vein, the aorta, the spinal cord or internal organs (such as lungs), which could cause major complications if damaged during the surgery. Another drawback of laparoscopy is to provide only two-dimensional visualization to the surgeon while he is performing complex three-dimensional manipulations. 
To overcome these difficulties, surgeons have to be trained more intensively and more often. Many computer vision works in the field of laparoscopic surgery had, therefore, focused on improving training procedures. Thus, surgery simulation software has been developed to artificially reproduce surgical conditions such as bleeding, force feedback, tissue deformations, etc. ([1],[2], [3] and references therein for example).

Another possible way to overcome the difficulties of laparoscopic surgery is to develop computer vision software to assist surgeons during the surgeries. With computer vision methods it would be possible to compute depth maps, to do partial 3D reconstructions of some anatomical structures, to register live 3D models with preoperative data in order to assist the surgery in real time, etc. ([4] ,[5], [6] to cite only few).

However complex computer vision systems cannot be developed without solving some prerequisite problems. In this paper, an approach is presented to solve one of those problems : the segmentation of laparoscopic images.

The main objective of this paper is to extract the surgical instruments inserted during the surgery from the recorded images. This extraction is important because anatomical structures are prone to exhibit non-rigid motion (because of their elastic nature) and surgical instruments are usually rigid. Thus, tracking geometric features of the instrument(s) will allow the self-calibration of the image sequence. Wei et al. [7] and Zhang et al. [8] tried to track surgical instruments in laparoscopic images but their methods rely on markers, which is not suitable in practice. The proposed method extracts the instrument(s) without using predefined markers.

\section{Proposed Approach}

The developed approach requires three steps. First of all, input images are preprocessed by a homomorphic filter. The preprocessing phase is needed because of the large variability in lighting and focus conditions. The output of the preprocessing is then used to compute the initial segmentation map. Finally, the obtained regions are classified as being part of the background (which includes the anatomical structures) or part of an instrument.

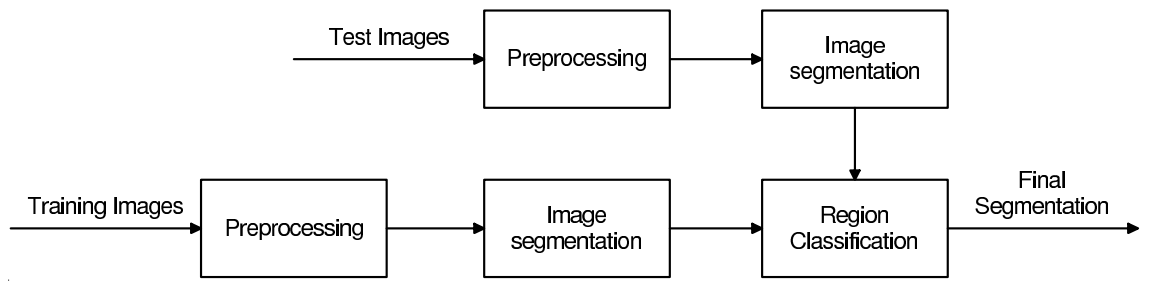

Fig. 1. Block diagram: Instrument segmentation 


\subsection{Image Acquisition System and Hardware}

The acquisition system is composed of an endoscope connected to a digital recording system both from Stryker Endoscopy. The recorded images are either 640x480x24bits (still images) or 320x240x24bits (images extracted from a video sequence).

Endoscopes are optical devices characterized by small lenses (typically less than $10 \mathrm{~mm}$ ), which results in different kinds of image distortions. Also, to reduce the number of incisions, light source and camera head are merged in a single unit. Therefore, the light comes from a point located on the optical axis and close to the imaged structures. This particular configuration caused many problems, such as variable illumination of the scene and specular reflexion causing pixel saturation and information loss.

\subsection{Pre-processing Algorithm}

The preprocessing algorithm consists in a homomorphic filtering followed by histogram scaling. Homomorphic filters were chosen because they are well suited to handle variable illumination problems inherent to laparoscopic surgeries.

The basic idea of homomorphic filtering is to think about an image as the product of two signals: the reflectance and the illumination. Reflectance is determined by the characteristics of the imaged object and illumination is determined by the intensity and position of light source(s). A logarithmic transformation converts the product of two signals into an addition of two signals. A high pass filter can therefore be used to separate the high frequencies associated to the reflectance from low frequencies associated to illumination.

$$
\begin{gathered}
I(x, y)=i(x, y) r(x, y) \\
z(x, y)=\ln (I(x, y))=\ln (i(x, y))+\ln (r(x, y)) \\
z^{\prime}(x, y)=z(x, y) \otimes h(x, y) \\
I^{\prime}(x, y)=e^{z^{\prime}(x, y)}
\end{gathered}
$$

The interested reader can find a more complete introduction to homomorphic filtering in [9]. Because the results of the preprocessing are used by a segmentation algorithm and not by a human being, a simple filtering scheme can be chosen. Therefore, the filtering was done using the following equation.

$$
z^{\prime}(x, y)=z(x, y)-z(x, y) \otimes \frac{1}{2 \pi \sigma^{2}} e^{\frac{x^{2}+y^{2}}{2 \sigma^{2}}}
$$

The value of the parameter $\sigma$ was chosen empirically and set to 20. This value offers a good compromise between the elimination of the illumination variability inside the images and the preservation of reflectance details given the lighting and zoom conditions experienced during this experiment (a different value may be needed to produce good results with a different endoscope).

The homomorphic filtering usually compress the image histogram ; thus the intensity range of the filtered images is not fully used. To avoid this undesirable effect, the image histogram is linearly scaled after the homomorphic filtering. 


\subsection{Segmentation Algorithm}

The selected image segmentation algorithm has to produce an initial segmentation map, which will be used by the classification module to determine the subsets of regions belonging to surgical instrument(s) or to the background. Moreover, the initial segmentation has to be totally automated, thus methods based on manual initialization (like geodesic active contours [10], for instance) are not suitable.

Thus, an image segmentation algorithm based on the JSEG criteria [11] has been chosen to perform the initial segmentation. This segmentation algorithm described by Y. Deng and B.S. Manjunath in [11] has three important parameters: a threshold $t_{q}$ that controls the color quantification, the number of scales $n_{s}$ used and another threshold $t_{m}$ that controls the region merging process. The method is quite robust with respect to the particular values of the parameters $t_{q}, t_{m}$ and $n_{s}$. Therefore the values were selected empirically for each image set and remain constant for all the input images within an image set.

\subsection{Region Classification}

The proposed system uses Support Vector Machines (SVMs) [12] to classify the regions found by the initial segmentation algorithm. The choice of SVMs is mainly justified by the fact that SVMs minimize a bound on the structural risk (the probability of misclassifying previously unseen data) instead of the empirical risk (misclassification on the training set). Thus good generalization performance is usually achieved when only a limited amount of data is available for training, which is often the case in the medical field. SVMs can also easily handle different kinds of nonlinear decision frontiers by selecting a suitable kernel function. However other nonparametric classifiers such as K-NN or multiple layers neural networks (using regularization or/and pruning) could also be used.

The features selected for the region classification are based on color distribution or region geometry. Color distribution features selected are the twodimensional normalized color histograms computed from standard chromaticity coordinates $\left(r^{\prime}=R /(R+G+B)\right.$ and $\left.g^{\prime}=G /(R+G+B)\right)$ and the pixel intensity histogram both computed for each region. The two histograms are computed using 256 bins ( 16 bins X 16 bins for the two-dimentional normalized color histogram). Thus, 512 features were used to characterize color distribution of the regions. The utilization of histograms for classification came from the fact that this family of features has shown good results for image classification ([13], [14]). The selected geometric features are the first two Hu's moments [15]. It is useful to consider geometric features because illumination, geometry and material characteristics of surgical instruments often create recognizable region shapes.

\section{Experimental Results}

In order to assess the performance of the proposed method, two experiments have been conducted using two image sets. The ground truth was computed using 
manual segmentation. Training was performed using two thirds of the images in each set and the remaining third was used for testing. The distributions of the chosen features make linear classification difficult. Thus, a radial basis function (RBF) kernel with $\gamma=1.0$ was used to avoid this problem.

The first experiment was done on a set of 21 laparoscopic images. In this experiment, the background is either a wooden table or a green paperboard. The parameters of the JSEG method chosen for this image set were $t_{q}=250$, $t_{m}=0.4$ and $n_{s}=2$.

The second experiment was done on a set of 87 images recorded during laparoscopic surgeries performed at Sainte-Justine Hospital of Montreal. The images were acquired during two surgeries performed on scoliotic patients and one surgery performed on a minipig (the usual animal model for that kind of surgery). During this experiment, the chosen parameters of the JSEG method were $t_{q}=100, t_{m}=0.4$ and $n_{s}=2$.

The first experiment resulted in a pixel classification error rate of $2.5 \%$ (which correspond to a region classification error rate of $7.1 \%$ ). The second experiment (images from real surgeries) resulted in a pixel classification error rate of $13.1 \%$ (which correspond to a region classification error rate of 14.8\%). Figure 2 and figure 3 are segmentation examples from the first and from the second image set respectively.

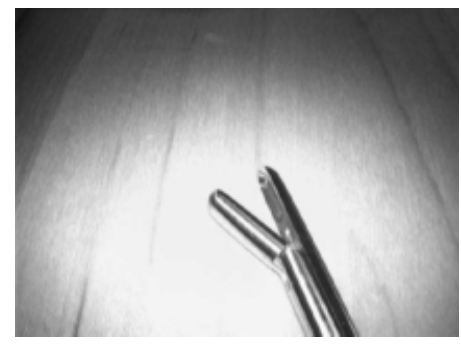

(a)

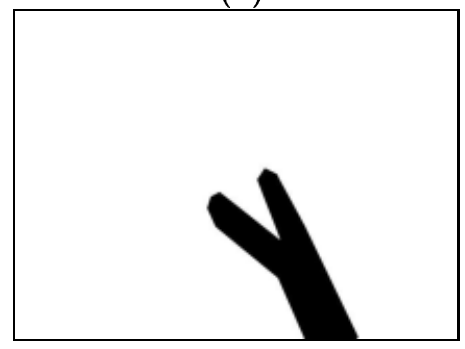

(c)

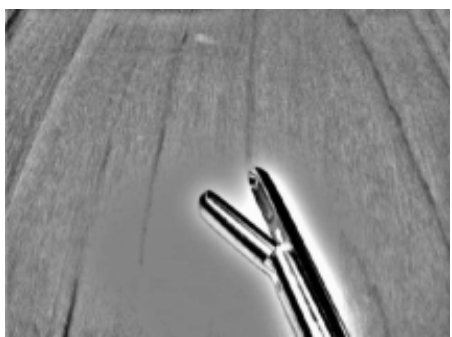

(b)

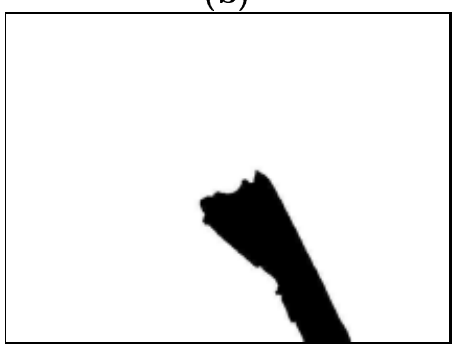

(d)

Fig. 2. Results of the instrument segmentation of an image with homogeneous background. (a) Original image. (b) Preprocessed image. (c) Manual segmentation of the instrument. (d) Result of the automatic segmentation. 


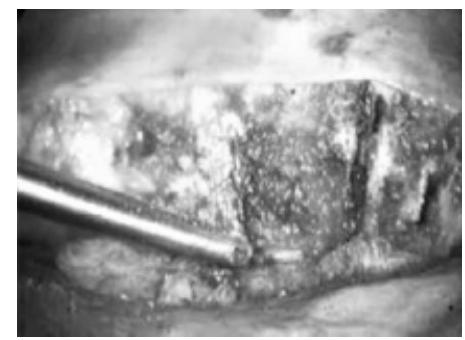

(a)

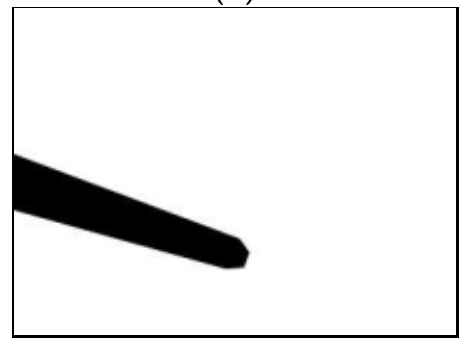

(c)

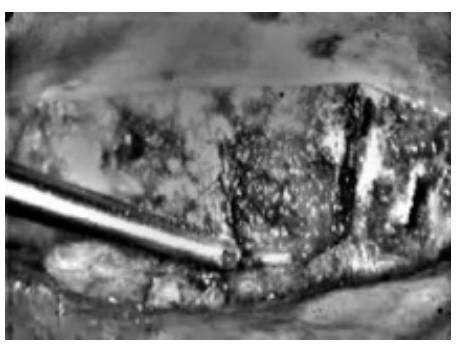

(b)

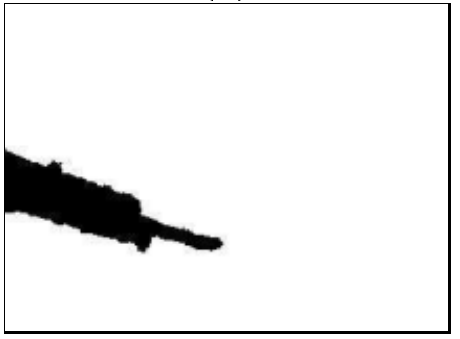

(d)

Fig. 3. Results of the instrument segmentation of a real surgery image. (a) Original image. (b) Preprocessed image. (c) Manual segmentation of the instrument. (d) Result of the automatic segmentation.

\section{Discussion}

In this method, the regions created by the image segmentation algorithm are classified in order to test their membership to a predefined class of object of interest. The regions computed by the image segmentation algorithm are not modified. Thus the classification step can be regarded as a specialized region merging algorithm. Furthermore, the success of the method is greatly influenced by the relative amount of work done by the image segmentation algorithm. For example, an image largely oversegmented by the image segmentation algorithm would certainly fail at the classification step because the region's histograms would not be representative of the object of interest or representative of one of its regions. On the other hand, an image segmented using too few regions by the image segmentation algorithm would not be useful either because it is likely to include regions containing both background and object of interest pixel's.

This dependence to the initial segmentation probably explains the difference between the results of the two experiments. The cluttered backgrounds, the proportion of saturated pixels and partial occlusions of the instrument(s) present on the images acquired during real laparoscopic surgeries caused lower quality initial segmentations, which failed to be correctly classified. 
Moreover, the classification module does not take into account the spatial relationships between the regions (such as the presence of an edge at the frontier between two regions) to compute the final segmentation map. Also, the classification module does not consider topological constraints on the final segmentation. The addition of a feedback mechanism between the classification and the image segmentation modules may be a solution to those problems.

Specular reflexion causing pixel saturation is another source of error in the segmentation and classification steps. This problem could be addressed in the preprocessing module using techniques such as inpainting [16], [17].

The nature and the parameters of kernel used for the region classification were both chosen empirically. However a more specialized kernel may lead to better results. Furthermore, the kernel parameter(s) may be automatically selected using optimization techniques.

Finally, the proposed method used binary classification (one class for the background and the other for the surgical instrument(s) ). However, the method could easily be extended to multiclass applications by using one-against-all or pairwise classification strategies for instance.

\section{Conclusion}

In this paper, a method to segment laparoscopic images using a combination of a region growing image segmentation algorithm and SVMs based classification has been presented and tested. The results of the segmentation on two different image sets have also been presented. One of the objectives of this study was to demonstrate the feasibility of segmenting surgical instruments in laparoscopic images without using markers. Experimental results suggest that this approach is feasible.

Also, the applicability of the developed method is not limited to laparoscopic images; it can be used in other application fields where the results of fully automated segmentation algorithms have to be reinterpreted to obtain a segmentation suitable for application purposes. Future work includes the development of self-calibration algorithm based on geometric features tracked through a sequence of surgical instrument images. It will then be possible to test the method in a more realistic application environment.

\section{Acknowledgement}

This work was supported in part by a grant from the Natural Sciences and Engineering Research Council of Canada.

\section{References}

1. Kuhnapfel, U., Cakmak, H., Maass, H.: Endoscopic surgery training using virtual reality and deformable tissue simulation. Computers \& Graphics UK 24 (2000) 671-682 
2. Cotin, S., Delingette, H., Ayache, N.: Real-time elastic deformations of soft tissues for surgery simulation stéphane cotin, hervé delingette, and nicholas ayache. IEEE Trans. on Visualization and Computer Graphics 5 (1999) 62-73

3. Basdogan, C., Ho, C., Srinivasan, M.: Simulation of tissue cutting and bleeding for laparoscopic surgery using auxiliary surfaces. In: Proc. Medicine Meets Virtual Reality, IOS Press (1999) 38-44

4. Dey, D., Gobbi, D., Slomka, P., Surry, K., Peter, T.: Automatic fusion of freehand endoscopic brain images to threedimensional surfaces: Creating stereoscopic panoramas. IEEE Trans. on Medical Imaging 21 (2002) 23-30

5. Bricault, I., Ferretti, G., Cinquin, P.: Registration of real and ct-derived virtual bronchoscopic images to assist transbronchial biopsy. IEEE Trans. on Medical Imaging 17 (2002) 703-714

6. Lunn, K., Hartov, A., Roberts, D., Sun, H., Paulsen, K.: Extracting displacement data from coregistered ultrasound for brain modeling. In: Proc. of the SPIE Medical Imaging. (2002)

7. Wei, G., Arbter, K., Hirzinger, G.: Real-time visual servoing for laparoscopic surgery. controlling robot motion with color image segmentation. IEEE Engineering in Medicine and Biology Magazine 16 (1997) 40-45

8. Zhang, X., Payandeh, S.: Application of visual tracking for robot-assisted laparoscopic surgery. Journal of Robotic Systems 19 (2002) 315-328

9. Gonzalez, R., Woods, R.: Digital Image Processing. Prentice Hall (2002)

10. Caselles, V., Kimmel, R., Sapiro, G.: Geodesic active contours. International Journal of Computer Vision 22 (1997) 61-79

11. Deng, Y., Manjunath, B.: Unsupervised segmentation of color-texture regions in images and video. IEEE Trans. on Pattern Analysis and Machine Intelligence 23 (2001) 800-810

12. Vapnik, V.: The Nature of Statistical Learning Theory. Springer-Verlag (1995)

13. Chapelle, O., Haffner, P., Vapnick, V.: Support vector machines for histogrambased classification. IEEE Trans. on Neural Networks 10 (1995) 1055-1064

14. Tsutsumi, F., Nakajima, C.: Hybrid approach of video indexing and machine learning for rapid indexing and highly precise object recognition. In: Proc. ICIP. Volume 2. (2001) 645-648

15. Hu, M.K.: Visual pattern recognition by moment invariants. IRE Trans. Information Theory 8 (1962) 179-187

16. Bertalmio, M., Sapiro, G., Caselles, V., Ballester, C.: Image inpainting. In: Siggraph 2000, Computer Graphics Proceedings, ACM Press / ACM SIGGRAPH / Addison Wesley Longman (2000) 417-424

17. Ballester, C., Bertalmio, M., Caselles, V., Sapiro, G., Verdera, J.: Filling-in by joint interpolation of vector fields and grey levels. IEEE Trans. on Image Processing 10 (2000) 1200-1211 